\title{
Study of the geometrical resonances of superconducting tunnel junctions
}

\author{
Sørensen, O. Hoffmann; Finnegan, T.F.; Pedersen, Niels Falsig
}

Published in:

Applied Physics Letters

Link to article, DOI:

$10.1063 / 1.1654583$

Publication date:

1973

Document Version

Publisher's PDF, also known as Version of record

Link back to DTU Orbit

Citation (APA):

Sørensen, O. H., Finnegan, T. F., \& Pedersen, N. F. (1973). Study of the geometrical resonances of superconducting tunnel junctions. Applied Physics Letters, 22(4), 129-131. https://doi.org/10.1063/1.1654583

\section{General rights}

Copyright and moral rights for the publications made accessible in the public portal are retained by the authors and/or other copyright owners and it is a condition of accessing publications that users recognise and abide by the legal requirements associated with these rights.

- Users may download and print one copy of any publication from the public portal for the purpose of private study or research.

- You may not further distribute the material or use it for any profit-making activity or commercial gain

- You may freely distribute the URL identifying the publication in the public portal

If you believe that this document breaches copyright please contact us providing details, and we will remove access to the work immediately and investigate your claim. 


\title{
Study of the geometrical resonances of superconducting tunnel junctions*
}

\author{
O. Hoffmann Soerensen, T.F. Finnegan ${ }^{\dagger}$, and N.F. Pedersen \\ Physics Laboratory I, The Technical University of Denmark, DK-2800 \\ Lyngby, Denmark \\ (Received 18 July 1972)
}

\begin{abstract}
The resonant cavity structure of superconducting $\mathrm{Sn}-\mathrm{Sn}$-oxide-Sn tunnel junctions has been investigated via photon-assisted quasiparticle tunneling. We find that the temperature-dependent losses at $35 \mathrm{GHz}$ are determined by the surface resistance of the Sn films for reduced temperatures between 0.5 and 0.8 . Our results are in very good agreement with the microscopic theory of Mattis and Bardeen for the surface resistance of Sn.
\end{abstract}

Electromagnetic waves can propagate in a superconducting tunnel junction with a phase velocity much smaller than the free-space velocity of light. ${ }^{1}$ The resonant structure associated with such a junction manifests itself in experiments involving the ac Josephson effect ${ }^{2-5}$ and photon-assisted tunneling. ${ }^{6,7}$ These cavity modes play a particularly crucial role in high-precision measurements of $2 e / h$ (via the microwave-induced steps) made with the use of Josephson tunnel junctions. ${ }^{8} \mathrm{Al}$ though the existence of these modes has been well established, the nature of the losses determining the linewidth or $Q$ has not been studied experimentally. Much interest in the various loss mechanisms has recently developed of the Josephson quasiparticle-pair interference current by Pedersen, Finnegan, and Langenberg. ${ }^{9}$ To clarify the role of this interference term of the ac properties of a junction, it is essential to understand the other junction loss mechanisms. We report here the first detailed measurements of the geometrical resonance linewidth or $Q$. By comparing the experimental results with calculations based on an equivalent circuit representation of the junction similar to that of Scott, ${ }^{10}$ we find that the dominant loss mechanism for reduced temperatures above 0.5 can be attributed to the surface resistance of the superconducting films which form the junction.

For a one-dimensional tunnel junction, the fundamental resonant frequency $\omega_{R}=\pi \bar{c} / L$ is determined by the junc tion length $L$ and the phase velocity $\bar{c}$. The latter quantity is a function of the penetration depth $\lambda$ of the electromagnetic fields, and $\bar{c}$ is therefore both temperature and frequency dependent.

For high microwave frequencies, a particularly convenient way to study the geometrical resonance is to vary $\omega_{R}$ by means of the temperature. The use of the photonassisted tunneling phenomenon provides a simple and direct method for investigating the mode structure.

Hamilton and Shapiro ${ }^{6}$ have shown that when a junction is coupled to external microwave fields, standing waves will be set up in the junction and therefore must be included in the theoretical description of the problem. Recently, Soerensen and Samuelsen ${ }^{7}$ have shown that the relative step height, $a_{N}$, of the $N$ th photon-assisted step, when the applied frequency $\omega=n \omega_{R}$, is

$$
a_{N}=(1 / L) \int_{0}^{L} J_{N}^{2}(\alpha) d x,
$$

with $\alpha=e v_{\mathrm{rt}}(x) / \hbar \omega$, where the rf voltage across the junction has the form

$$
v_{\mathrm{rP}}(x)=v_{0} \cos (n \pi x / L) .
$$

The $a_{N}$ are not very sensitive to the detailed spatial variation of $v_{\mathrm{rf}}(x)$ near $\omega=n \omega_{R}$, and for $N=0$ and $N=1$ [i.e., the steps at $2 \Delta / e$ and at $(2 \Delta-\hbar \omega) / e$ ] the dependence of the relative step amplitudes on $\left\langle\alpha^{2}\right\rangle$ is nearly independent of the spatial variation for small values of $v_{0} \cdot{ }^{11}$ (The brackets \langle\rangle denote a spatial average.) Exact calculations of $a_{N}$ have been carried out by assuming the voltage dependence described by Eq. (2). ${ }^{7,11} \mathrm{Ex}-$ perimentally, a constant microwave field is coupled to the junction, and the observed height of the $N=0$ step, $a_{0}$, is used to determine $\left\langle\alpha^{2}\right\rangle$ and, hence, $\left\langle v_{\mathrm{r}}^{2}\right\rangle$ in the junction at various temperatures.

A tunnel junction can be regarded as a section of transmission line with an equivalent circuit as indicated in Fig. 1. The line is terminated at each end by an effective impedance $Z_{0}$ which phenomenologically represents the radiation losses at the edges of the junction. For simplicity we take the impedance to be purely resistive, i.e., $Z_{0}=R_{0} .{ }^{12}$ The relevant junction parameters are obtained by solving the Maxwell and London equations in the two-fluid model. For a tunnel junction with thick films, the resistance $r$, the inductance $l$, and the capacitance $c$ per unit length are, respectively,

$$
\begin{aligned}
& r=2 R_{s} / w, \\
& l=\mu_{0}(2 \lambda+d) / w, \\
& c=\epsilon_{0} \epsilon_{r} w / d .
\end{aligned}
$$

Here $R_{s}$ is the real part of the surface impedance while $\lambda$ is related to the imaginary part of the surface impedance $X_{s}$ by the relation $X_{s}=\omega \mu_{0} \lambda$. The remaining quantities are $\epsilon_{r}$, the relative dielectric constant of the oxide layer; $w$, the junction width; and $d$, the oxide thickness. The external microwave source appears as a constant current source coupled to the junction via the external waveguide impedance. The microwave currents induced at the edges of the junction will in general be a superposition of in-phase and out-of-phase components depending on whether the excited currents are in-phase or out-of-phase at the two junction edges. The symmetry of the external field is denoted even (in-phase) or odd (out-of-phase), corresponding to the parity of the junction mode numbers $n$ [see Eq. (2)] which can be excited.

Using the junction model shown in Fig. 1, we have

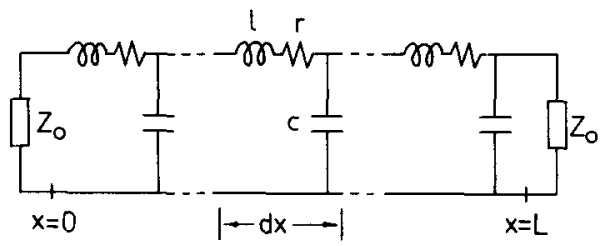

FIG. 1. Equivalent circuit of the tunnel junction strip line. 


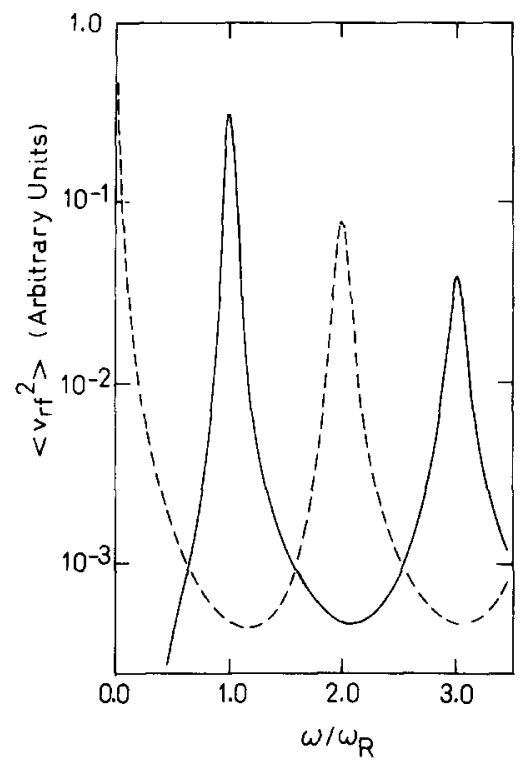

FIG. 2. Mean square of the microwave-induced voltage as a function of frequency for external fields of even symmetry (broken line) and odd symmetry (solid line) with $R_{s} / X_{s}=0.05$ corresponding to a reduced temperature $t=0.675$.

evaluated the voltage $v_{\mathrm{rf}}$ as a function of the coordinate $x$, the temperature, and the applied frequency. The microwave losses enter via the ratio $R_{s} / X_{s}$. This ratio was obtained from a calculation of the surface impedance $R_{s}+i X_{s}$ for $\mathrm{Sn}$ as a function of temperature and frequency with the use of the theory of Mattis and Bardeen. ${ }^{13}$ The calculations were made for the case of a finite mean free path $l$ and diffuse surface scattering. ${ }^{14}$ For $l=1000 \AA, R_{s} / X_{s}$ varies almost exponentially between 0.016 at a reduced temperature $t=0.50$ and 0.19 at $t=0.90$. (The reduced temperature $t$ equals the ratio $T / T_{c}$, where $T_{c}$ is the superconducting transition temperature.) Over this entire temperature range, $R_{s} / X_{s}$ for $\omega=\omega_{R}$ and $\omega=2 \omega_{R}$ are the same within $10 \%$.

In Fig. 2, the quantity $\left\langle v_{\mathrm{rt}}^{2}\right\rangle$ is shown plotted as a function of the applied frequency for a constant $t=0.675$. From this figure, it is clear that near frequencies $\omega^{\prime}=n \omega_{R}$ either an even or odd mode is sharply peaked, and therefore the resonant response does not depend critically on nonideal coupling to the external fields. The quality factor $Q$ of the junction resonance [defined by $Q=2 \pi$ (energy stored)/(energy dissipated per cycle)] can be expressed in terms of the junction parameters. If the film losses are dominant,

$$
Q=\omega l / r \approx X_{s} / R_{s} \text {. }
$$

The results of a series of measurements in the same junction are shown in Fig. 3. The observed $\left\langle v_{\mathrm{rf}}^{2}\right\rangle$ are plotted as a function of temperature for three different applied frequencies. The corresponding theoretical results obtained via the surface impedance calculations are also shown. The ratio between the barrier thickness and the dielectric constant, $d / \epsilon_{r}$, has been used as an adjustable parameter. The solid lines in Fig. 3 are the calculated results for $d / \epsilon_{r}=5.33 \AA$. This choice of $d / \epsilon_{r}$ gives the best over-all agreement with the experimental results and is consistent with $d \approx 20 \AA$ and $\epsilon_{r} \approx 4$. For each of the three cases shown in Fig. 3, the tempera- ture at which $\omega=\omega_{R}$ is indicated by an arrow.

As the applied frequency is increased, the peak position shifts toward lower temperatures. If the film losses are dominant, the amplitude of the induced $\mathrm{rf}$ voltage is proportional to $Q$, and, since $Q$ increases as $T$ decreases, the peaks in Fig. 3 become much sharper at lower temperatures. The agreement between the theoretical and experimental results is very good. We have also considered the effects of dielectric losses in the barrier in our calculations by varying the loss tangent and comparing the computed results with our experimental data. We have concluded that $\operatorname{Im} \epsilon_{r} / \operatorname{Re} \epsilon_{r}<5$ $\times 10^{-3}$. The $Q$ 's due to the losses in the films as determined from Eq. (4) were between 15 and 30 .

The junction resonant response near $\omega=2 \omega_{R}$ was also observed by using external frequencies of 69.28 and $69.42 \mathrm{GHz}$. The peaks occurred at temperatures in good agreement with those expected from theory by using the value of $d / \epsilon_{r}$ obtained at $\omega=\omega_{R}$ and by taking into account the dispersion due to the frequency dependence of $\lambda$. Evidence of a reproducible fine structure and a sharpening of the main peak was observed; however, because of experimental difficulties at these fre-

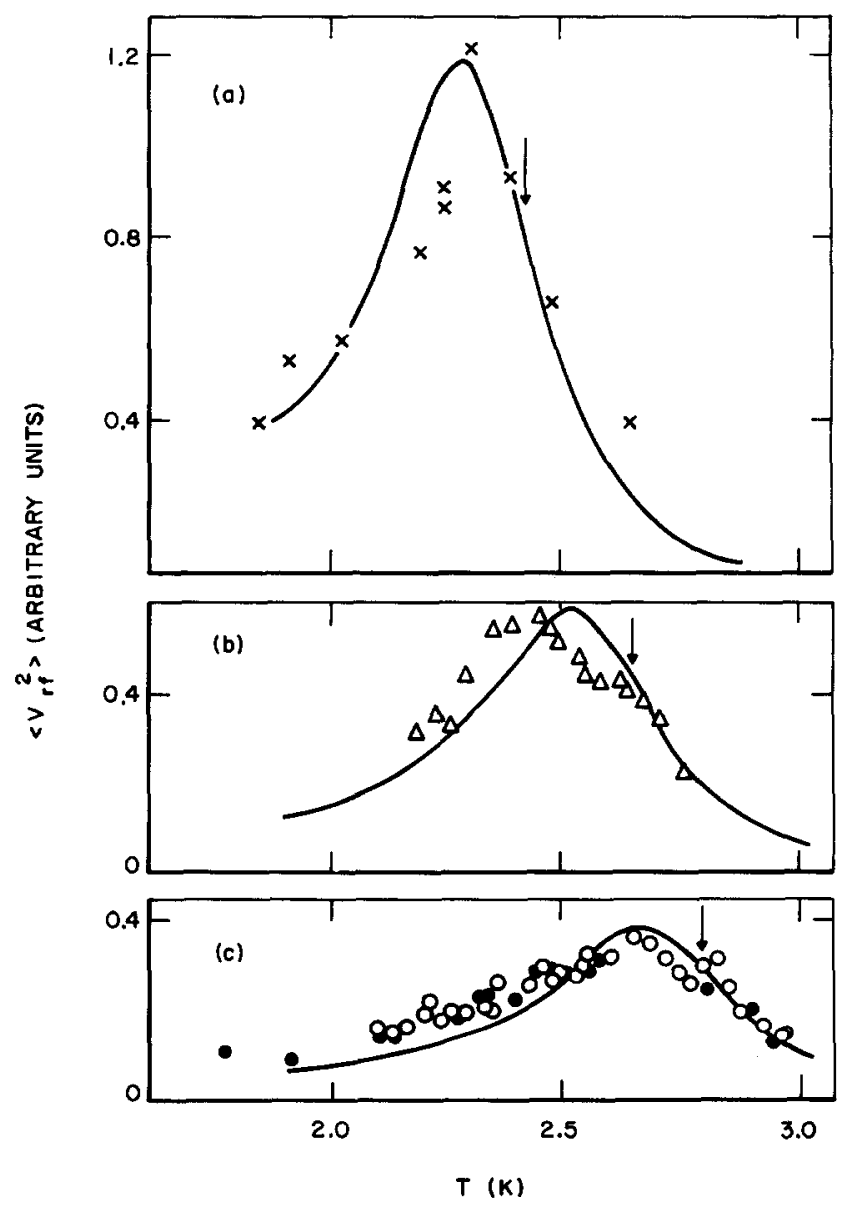

FIG. 3. Experimental and theoretical junction response as a function of temperature. The solid lines are theoretical results with junction parameters $L=0.26 \mathrm{~mm}, w=0.08 \mathrm{~mm}$, and $d / \epsilon_{r}=5.33 \AA$. (a) $f=34.70 \mathrm{GHz}$, external cavity at resonance; (b) $f=33.93 \mathrm{GHz}$, external cavity detuned; (c) $f=33.33$ $\mathrm{GHz}$, external cavity at resonance (full circles), external cavity detuned (open circles). The arrow in each case indicates the point $\omega_{R}(T)=\omega$. 
quencies, it is not meaningful to make a detailed comparison between theory and experiments. ${ }^{15}$

Some preliminary experiments on resonant $\mathrm{Pb}-\mathrm{Pb}$ oxide- $\mathrm{Pb}$ junctions at $4.2 \mathrm{~K}$ have been carried out by Finnegan and Toots ${ }^{16}$ using the microwave-induced Josephson steps to study the fundamental geometrical resonance $\left(\omega=\omega_{R}\right)$ at $9 \mathrm{GHz}$. In these experiments, the $Q$ was obtained by fitting a Lorentzian curve to the observed frequency-dependent response of the junctions to external microwave radiation. The observed Q's were about 110. Applying our theoretical model to a $\mathrm{Pb}$ tunnel junction (assuming a mean free path limited by the film thickness on the order of $1500 \AA$ ), we find $Q=150$, which is in rather good agreement with the observed results for $\mathrm{Pb}-\mathrm{Pb}$-oxide $-\mathrm{Pb}$ junctions.

In conclusion, we have shown that the high frequency losses in resonant superconducting tunnel junctions are dominated by losses in the superconducting films and not by losses in the dielectric oxide barrier. These losses in the films are adequately described by the surface impedance which is strongly temperature dependent. We believe that the dominant microwave losses in a Josephson tunnel junction are also due to the surface resistance of the superconducting films and therefore should have important consequences in furthering our understanding of the electrodynamics of Josephson junctions. Our results indicate it should be possible to control the $Q$ of a tunnel junction by using suitable superconducting alloys for the films.

The authors wish to thank U.K. Poulsen for carrying out the calculations of the surface impedance, and Professor K. Saermark for his very stimulating interest in this work. One of us (T.F.F.) wishes to thank Professor K. Saermark and Physics Laboratory I, The Technical University of Denmark for their hospitality during his stay, and Dr. B.N. Taylor and the National
Bureau of Standards for making the stay possible.

*Work supported in part by "Statens Naturvidenskabelige Forskningsrad" of Denmark.

'Permanent address: National Bureau of Standards, Washington, D.C. 20234. This work performed while on assignment at Physics Laboratory I, The Technical University of Denmark, Lyngby, Denmark.

${ }^{1} J$. C. Swihart, J. Appl. Phys. 32, 461 (1961).

${ }^{2}$ M.D. Fiske, Rev. Mod. Phys. 36, 221 (1964); D. D. Coon and M. D. Fiske, Phys. Rev. 138, A744 (1965).

${ }^{3}$ D. N. Langenberg, D.J. Scalapino, B.N. Taylor, and R.E. Eck, Phys. Rev. Letters 15, 294 (1965); 15, 842 (1965).

${ }^{4} \mathrm{~J} . T$. Chen, T.F. Finnegan, and D.N. Langenberg, Physica 55, 413 (1971).

${ }^{5}$ S. Bermon and R.M. Mesak, J. Appl. Phys. 42, 4488 (1971).

${ }^{6} \mathrm{C}$, A. Hamilton and S. Shapiro, Phys. Rev. B2, 4494 (1970).

${ }^{7} \mathrm{O}$. Hoffmann Soerensen and M.R. Samuelsen, Phys. Letters A 39, 137 (1972).

${ }^{8}$ T. F. Finnegan, A. Denenstein, and D. N. Langenberg, Phys. Rev. B 4, 1487 (1971).

${ }^{9}$ N. F. Pedersen, T. F. Finnegan, D. N. Langenberg, Phys. Rev. B 6, 4151 (1972); Proceedings of the XIII International Conference on Low Temperature Physics, edited by R. H. Kropschot and K.D. Timmerhaus (University of Colorado P. Boulder, Colo., to be published).

${ }^{10}$ A. C. Scott, Solid-State Electron. 7, 137 (1964).

${ }^{11}$ M. R. Samuelsen, B. Kofoed, and O. Hoffmann Soerensen, Phys. Status Solidi A 11, K79 (1972).

${ }^{12}$ The magnitude of $R_{0}$ may be estimated from the external waveguide impedance. In the case considered here, the radiation losses are small, compared to the internal losses, and may be neglected.

${ }^{13}$ D. C. Mattis and J. Bardeen, Phys. Rev. 111, 412 (1958).

${ }^{14}$ The reported values of the superconducting parameters for tin [found, e.g., in the review by J. Bardeen and J.R. Schrieffer in Progress of Low Temperature Physics, III, edited by C.J. Gorter (North-Holland, Amsterdam, 1961), p. 243] have been used. The mean free path $l$ was estimated from a measurement of the resistivity of the Sn films. The result for our junctions was $l \approx 1000 \AA$.

${ }^{15} \mathrm{At} 69 \mathrm{GHz}$, the waveguide system was oversized. Also, at these frequencies the sample heating was a serious problem.

${ }^{16}$ T. F. Finnegan and J. Toots (unpublished). 